\title{
A case report of Guillain-Barré syndrome in a pregnant woman infected by COVID-19
}

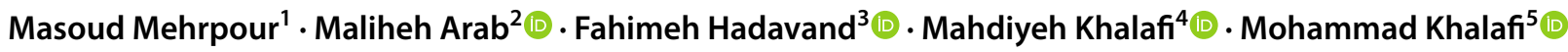

Received: 8 February 2021 / Accepted: 30 April 2021 / Published online: 3 June 2021

(c) Belgian Neurological Society 2021

Keywords COVID-19 $\cdot$ SARS-CoV-2 · Guillain-Barré Syndrome · Pregnancy

\author{
Abbreviations \\ COVID-19 Coronavirus disease 2019 \\ SARS-CoV-2 Severe acute respiratory syndrome corona- \\ virus 2 \\ CT Computed tomography \\ RT-PCR Reverse transcription-polymerase chain \\ reaction \\ ICU Intensive care unit
}

Sir,

Guillain-Barré syndrome (GBS) is a rare condition caused by the immune system attacking peripheral nerves resulting in the demyelination of nerve cells, manifested by muscle weakness and paresthesia [1]. People with GBS may be at higher risk of infection by COVID-19 than those in the general population. The present case reports on GBS as observed in a pregnant woman following a mild symptomatic COVID-19 infection.

In mid-November 2020, a 29-year-old pregnant woman presented with an initial complaint of tingling sensations in

Maliheh Arab

drmarab@sbmu.ac.ir

Masoud Mehrpour

mehrpr@yahoo.com

Fahimeh Hadavand

Fahimehadavand140@gmail.com

Mahdiyeh Khalafi

Khalafi8051@gmail.com

Mohammad Khalafi

Khalafi419@gmail.com

1 Imam Hossein Hospital, Shahid Beheshti University of Medical Sciences, Tehran, Iran

2 Department of Gynecology-oncology, Imam Hossein Medical Center, Imam Hossein Hospital, Shahid the distal aspect of all four limbs and mild weakness in the lower limbs that began the day before. The patient was a gravid 1, 18-19-week, pregnant woman. She had no history of any specific neurologic or rheumatic diseases and her vital signs were within the normal range. She had flu-like symptoms such as fever, chills, myalgia, headaches, and a runny nose which began about 10 days before her presentation but she had not seen a doctor due to her concerns about COVID-19 exposure during the pandemic while pregnant. She was reviewed by the gynecology and obstetrics team, and neurology services upon arrival in the emergency room. On examination, DTRs were absent and vibration sense was reduced in her lower limbs. There was no urinary retention and no spinal sensory level was detected. Muscle power was reduced to $2 / 5$ in her lower limbs, but remained normal in her upper limbs; the findings were suggestive of progressive symmetrical ascending weakness. Following the initial diagnosis of GBS, the patient was admitted to the intensive care unit and was scheduled to receive $0.4 \mathrm{~g} / \mathrm{kg} /$ day of IVIg. Before receiving the IVIg, blood samples were taken to perform the necessary tests. Nasopharyngeal and oropharyngeal samples were sent for SARS-CoV-2 polymerase chain reaction (PCR). EMG-NCV was ordered for this patient. On the second day of hospitalization the patient experienced dysphagia and nasal speech, with tachycardia (121/ min). Other vital signs were normal. SARS-CoV-2 PCR was reported as negative while anti-SARS-CoV-2 IgG and IgM

Beheshti University of Medical Sciences, Madani Street, Tehran 1617763141, Iran

3 Infectious Diseases and Tropical Medicine Research Center, Shahid Beheshti University of Medical Sciences, Tehran, Iran

4 Department of Obstetrics and Gynecology, Faculty of Medicine, Shahid Beheshti University of Medical Sciences, Tehran, Iran

5 Student's Research Committee, Tabriz University of Medical Sciences, Tabriz, Iran 
were reported as positive. SARS-CoV-2 IgG had an index of $7.2(<0.88$ is considered negative) and her IgM had an index of 7.1 ( $<0.98$ is considered negative). These results confirmed the diagnosis of COVID-19 infection. Chest computed tomography (CT) was not performed due to the absence of clinical signs and pregnancy. The EMG-NCV indicated damage to the myelin sheath of the peripheral nerves, causing a decrease in the speed of transmission of neural messages; this strengthened the possibility of GBS in the patient. On a no-contrast MRI of the brain, abnormalities such as nerve enhancement were not detected. No spinal thickening or enhancement was detected in the spinal MRI images. The patient received five intravenous immunoglobulin (IVIG) doses during her intensive care unit admission. During her hospital stay, the patient's general condition improved, her pulse increased to 84-95/min, and her muscle strength partially improved to $4 / 5$, after receiving the fifth IVIg dose. On ultrasound tests performed in the emergency room and before hospital discharge, a live fetus was seen with a variable presentation and a normal heart rate, the placenta was anterior and the amniotic fluid volume was normal. She was discharged after 7 days of hospitalization and was closely followed for 2 weeks after discharge.

Flu-like syndrome along with pulmonary parenchymal involvement in a chest CT scan are some of the findings reported in COVID-19 patients developing GBS. Autoimmune cross-reactivity between viral structure and neuronal component in the PNS seems to be the main reason for the occurrence of GBS in viral infections [2].

Hukuimwe and co-workers reported GBS in a pregnant woman following an upper respiratory tract infection [3]. Zika virus rarely result in GBS [4]. Despite being a rare condition, GBS can result in life-threatening complications such as respiratory failure, which highlights the importance of early diagnosis and initiation of treatment, especially in pregnant women. High levels of estrogen during pregnancy are among the causes of an increase in the number of immune cells and, altering their function by acting as an immune modulator. Thus, pregnant women seem to have modulated immune systems compared to non-pregnant patients; this can be of significance when discussing GBS in pregnant women. While issues like dysphagia and quadriplegia are concerning complications of GBS, respiratory failure is considered the most lifethreatening complication, necessitating urgent attention and close monitoring. On the other hand, peak expiratory flow (PEF) increases significantly in pregnant women, and should be interpreted cautiously when used to monitor respiratory function in pregnant women suffering from GBS, especially in the presence of chest infections [5].

Besides supportive therapies like ICU monitoring, IVIg, and plasma exchange are used for GBS treatment, but the use of corticosteroids in studies has shown no significant adverse effect on the treatment outcome [6].
It seems that many of the complications and chronic problems caused by the COVID-19 pandemic are still unknown. GBS is one of these complications, which has the potential to cause respiratory failure; this necessitates early diagnosis and treatment of the condition, especially in pregnant women.

Acknowledgements Thanks to Prof. Jennifer Swann for editing in native English. The authors gratefully acknowledge and in memory of all medical staff, as well as thousands of unsung heroes participate in the frontline in the fight against the epidemic of SARS-CoV-2.

Author contributions All authors read and approved the final manuscript. All authors take responsibility for the integrity of the data and the accuracy of the data analysis.

\section{Funding None.}

Data availability The data used in this study are available from the corresponding author on request.

\section{Declarations}

Conflict of interest The authors declare that they have no competing interests.

Ethical approval This article does not contain any studies with human participants or animals performed by any of the authors.

Informed consent The patient informed consent has been obtained.

Consent for publication By submitting this document, the authors declare their consent for the final accepted version of the manuscript to be considered for publication.

\section{References}

1. Lawn ND, Wijdicks EF (1999) Fatal Guillain-Barré syndrome. Neurology 52(3):635-635

2. Abu-Rumeileh S, Abdelhak A, Foschi M, Tumani H, Otto M (2020) Guillain-Barré syndrome spectrum associated with COVID-19: an up-to-date systematic review of 73 cases. J Neurol 1:1-38

3. Hukuimwe M, Matsa TT, Gidiri MF (2017) Guillain-Barré syndrome in pregnancy: a case report. Womens Health 13(1):10-13

4. Pacheco LD, Saad AF, Hankins GD, Chiosi G, Saade G (2016) Guillain-Barré syndrome in pregnancy. Obstet Gynecol 128(5):1105-1110

5. Grindheim G, Toska K, Estensen ME, Rosseland L (2012) Changes in pulmonary function during pregnancy: a longitudinal cohort study. BJOG Int J Obstet Gynaecol 119(1):94-101

6. Hughes RA, Brassington R, Gunn AA, van Doorn PA (2016) Corticosteroids for Guillain-Barré syndrome. Cochrane Database Syst Rev 10:1

Publisher's Note Springer Nature remains neutral with regard to jurisdictional claims in published maps and institutional affiliations. 